\title{
Weighted-Lasso for Structured Network Inference from Time Course Data
}

\author{
Camille Charbonnier, Julien Chiquet, Christophe Ambroise. \\ Laboratoire Statistique et Génome \\ 523, place des Terrasses de l'Agora \\ 91000 Évry, FRANCE \\ e-mail: julien.chiquet@genopole.cnrs.fr; camille.charbonnier; christophe.ambroise \\ url: http://stat.genopole.cnrs.fr
}

\begin{abstract}
We present a weighted-LASsO method to infer the parameters of a first-order vector auto-regressive model that describes time course expression data generated by directed gene-to-gene regulation networks. These networks are assumed to own prior internal structures of connectivity which drive the inference method. This prior structure can be either derived from prior biological knowledge or inferred by the method itself. We illustrate the performance of this structure-based penalization both on synthetic data and on two canonical regulatory networks, first yeast cell cycle regulation network by analyzing Spellman et al's dataset and second E. coli S.O.S. DNA repair network by analysing U. Alon's lab data.
\end{abstract}

Keywords and phrases: Biological networks, Vector auto-regressive model, Lasso.

\section{Introduction}

Along the dozen of years of statistical studies related to microarrays for gene expression profiling, conditional dependency has been recognized as an appropriate statistical tool to model direct interactions between genes. Graph representation suits well such relationships between variables. As a consequence GGMs (Gaussian Graphical Models) have been widely studied by statisticians, particularly those looking for applications to the reconstruction of gene-to-gene regulation networks (See e.g. Schäfer and Strimmer 2005, Meinshausen and Bühlmann 2006, Wille and Bühlmann 2006, Castelo and Roverato 2006, Drton and Perlman 2007, Shimamura et al. 2007). In the context of transcriptomic data, the main statistical issue paradoxically relies on the scarcity of data: despite a shrinking cost, microarrays still provide dataset that fall into the high-dimensional setting. Namely, the number of variables (the $p$ genes) remains greater than the sample size $n$ (the number of microarray slides).

In the Gaussian independent identically distributed (hereafter i.d.d.) setting, each microarray experiment is considered as a realization of a Gaussian vector whose dependency structure is fully determined by its covariance matrix. It can be shown that non-null conditional dependencies between genes are described by nonzero entries of the inverse of the covariance matrix (Dempster 1972). Thus, inferring this matrix is equivalent to recovering the graph of interest, which is not trivial when $n$ is smaller than or of the order of $p$. To handle 
the data scarcity, methods based upon $\ell_{1}$-norm are very popular: they answer to both questions of regularization and of variable selection by selecting the most significant edges between genes in the network. In the i.i.d setting, $\ell_{1}$ penalized maximum likelihood Gaussian covariance estimation has been first investigated by Yuan and Lin (2007) and Banerjee et al. (2008) independently. These methods provide sparse graph estimates, sparsity being a characteristic of gene-to-gene regulation networks.

Looking for an improvement of these methods regarding the biological context, we provided in Ambroise et al. (2009) a method that not only looks for sparse solutions, but also for an internal structure of the network that drives the inference. Indeed, biological networks and particularly gene regulation networks are known not only to be sparse, but also organized, so as nodes belong to different classes of connectivity. Thus, we suggested a criterion that takes this heterogeneity into account. This leads to a better inference when networks are highly structured. Note that Marlin et al. (2009) published subsequently an independent paper providing a similar method in a Bayesian framework. In these two papers, the internal structure considered relies on affiliation networks. That is, genes are clustered into groups that share the same connectivity patterns. This can be seen as the analogous to the group-LAsso (Yuan and Lin 2006) applied to a graphical context.

Finally, some authors (e.g. Opgen-Rhein and Strimmer 2007, Lèbre 2009, Shimamura et al. 2009) underlined that transcriptomic dataset are not i.i.d. when considering time course expression data. Assuming a first-order vector auto-regressive (VAR1) model for the time course data generation, they provided inference methods handling high-dimensional settings: Opgen-Rhein and Strimmer suggested a shrinkage estimate while Lèbre performed statistical tests on limited-order partial correlations to select significant edges. In a recent work, Shimamura et al. (2009) proposed to deal with this VAR1 setup by combining ideas from two major developments of the LASso to define the Recursive elasticnet. As an elastic-net (Zou and T. 2005), this method adds an $\ell_{2}$ penalty to the original $\ell_{1}$ regularization, thus encouraging the simultaneous selection of highly correlated covariates on top of the automatic selection process due to the $\ell_{1}$ norm. As in the adaptive LASso (Zou 2006), weights are corrected on the basis of a former estimate so as to adapt the regularization parameter to the relative importance of coefficients. Note that, in this context, we are no longer looking for an estimate of the inverse of the covariance matrix but of the parameters of the VAR1 model, which leads to a directed graph.

In this paper, we aim to couple the time course data modeling by the VAR1 model to an $\ell_{1}$-regularizing approach that takes the internal structure of the network into account. This internal structure does not rely on an affiliation structure anymore since graphs inferred from time course data display a completely asymmetrical pattern. The internal structure adopted here splits the genes into two groups: a group of hubs that exhibit a high connection probability to all other genes and a group of leaves that only receive edges leaving from the hub class. This information can either be inferred as seen in this paper or recovered from biological expertise since recovering hubs consists roughly in 
exhibiting transcription factors in regulatory networks, a large number of them being already identified by the biologists.

Another refinement of our method is to built on the adaptive-LAsso (Zou 2006, Zhou et al. 2009) which is known to reduce false positive rate compared to the classical LAsso. As such, our method belongs to the larger family of weighted-LAsso methods. Shimamura et al. (2007) built upon Meinshausen and Bühlmann's neighborhood selection and the adaptive LASso to improve inference of networks in an i.i.d. context. They chose separate penalties for each node's neighborhood selection problem and adapted each individual penalty coefficient to the information brought by an initial ridge estimate. Here, we suggest to lower the bias of the LASSO by not only using information from an initial statistical inference but also from prior knowledge about the topology of the network that assumes the existence of genes with high connection probability to other genes.

The rest of the paper is organized as follows: in the next Section, the VAR1 model and the associated likelihood function are briefly recalled; an $\ell_{1}$-penalized criterion is proposed where each parameter of the VAR1 model, representing the graph of interest, is weighted according to its belonging to the hub group. The weights can also depend on a previous estimate just as in the adaptive-LAsso. We also briefly recall available tools to guide the choice of the regularization parameter. In Section 3, the inference procedure is detailed: we present how the internal structure can be recovered; from that point, network inference reduces to a convex optimization problem which we solve through an active-set algorithm based upon the approach of Osborne et al. (2000). Finally, an experimental Section investigates the performances of the method. First, simulated data are considered; then, we try to recover edges implied in two different regulation processes. First in yeast cell cycle, by analyzing the Spellman's dataset and comparing the selected edges to the direct regulations collected from the Yeastract database; second in E. coli, by analyzing U. Alon's precise kinetic data on S.O.S. DNA repair subnetwork.

Remark. The code will soon be embedded in the $\mathrm{R}$ package SIMoNe. (Chiquet et al. 2009).

\section{Modeling Heterogeneous Regulation Networks from Time Course Data}

\subsection{Auto-regressive Model and Sparse Networks}

Let $\mathcal{P}=\{1,2, \ldots, p\}$ be the set of variables of interest, e.g., some $p$ genes. Let us denote by $\left(X_{t}\right)_{t \in \mathbb{N}}$ the $\mathbb{R}^{p}$-valued stochastic process that represents the discretetime evolution of the gene expression levels, written as a row vector. Also denote by $X_{t}^{i}$ the expression level of gene $i$ at time $t$ and $X_{t}^{\backslash i}$ the expression level of all genes but $i$ at time $t$. Herein, $X_{t}$ is assumed to be generated by a first-order vector auto-regressive (VAR1) model

$$
X_{t}=X_{t-1} \mathbf{A}+\mathbf{b}+\varepsilon_{t}, \quad t \in \mathbb{N}^{*},
$$


where $\mathbf{A}=\left(A_{i j}\right)_{i, j \in \mathcal{P}}$ is an $p \times p$ matrix, $\mathbf{b}$ is a size- $p$ row vector and $\varepsilon_{t}$ is a white Gaussian process. Namely, $\varepsilon_{t} \sim \mathcal{N}(0, \mathbf{D})$ where $\mathbf{D}$ is a diagonal matrix such as $\mathbf{D}_{i i}=\sigma_{i}^{2}$ and $\operatorname{cov}\left(\varepsilon_{t}, \varepsilon_{s}\right)=\mathbf{1}_{\{s=t\}} \mathbf{D}$ for all $s, t>0$. Moreover, $X_{0} \sim \mathcal{N}(\mu, \boldsymbol{\Sigma})$, with $\mu$ a size- $p$ vector of means and $\boldsymbol{\Sigma}$ a covariance matrix. Also assume that $\operatorname{cov}\left(X_{t}, \varepsilon_{s}\right)=0$ for all $s>t$ : hence, $X_{t}$ is obviously a first-order Markov process.

Since the covariance matrix $\mathbf{D}$ is diagonal, each entry $A_{i j}$ is directly linked to the partial correlation coefficient between variables $X_{t}^{i}$ and $X_{t-1}^{j}$. In fact,

$$
A_{i j}=\frac{\operatorname{cov}\left(X_{t}^{j}, X_{t-1}^{i} \mid X_{t-1}^{\backslash i}\right)}{\operatorname{var}\left(X_{t-1}^{i} \mid X_{t-1}^{\backslash i}\right)},
$$

thus nonzero entries of $\mathbf{A}$ code for the adjacency matrix of a directed graph describing the conditional dependencies between the elements of $\mathcal{P}$. Inferring $\mathbf{A}$ is equivalent to reconstructing this graph and is the main issue of this paper.

To this end, let us set up the estimation framework: assume that $X_{t}$ is observed on the time space $t=0,1, \ldots, n$. Denote by $\mathbf{X}$ the $(n+1) \times p$ matrix of available centered, scaled to unit-variance data, whose $t^{\text {th }}$ row contains the information relative to the $p$ variables at time $t$. The empirical variance-covariance matrix $\mathbf{S}$ and the empirical temporal covariance matrix $\mathbf{V}$ are then given by

$$
\mathbf{S}=\frac{1}{n} \mathbf{X}_{\backslash n}^{\top} \mathbf{X}_{\backslash n}, \quad \mathbf{V}=\frac{1}{n} \mathbf{X}_{\backslash n}^{\top} \mathbf{X}_{\backslash 0},
$$

where $\mathbf{X}_{\backslash k}$ denotes matrix $\mathbf{X}$ deprived of its $k^{\text {th }}$ row.

The well-known maximum likelihood estimator (MLE) of $\mathbf{A}$ is easily recovered and recalled in the following proposition.

Proposition 1. Maximizing the log-likelihood of the VAR1 process is equivalent to the following maximization problem

$$
\max _{\mathbf{A}}\left\{\operatorname{Tr}\left(\mathbf{V}^{\boldsymbol{\top}} \mathbf{A}\right)-\frac{1}{2} \operatorname{Tr}\left(\mathbf{A}^{\top} \mathbf{S} \mathbf{A}\right)\right\},
$$

whose solution is given by

$$
\widehat{\mathbf{A}}^{\text {mle }}=\mathbf{S}^{-1} \mathbf{V} .
$$

Remark. Thanks to the assumptions we made on $\varepsilon$ the VAR 1 model can be seen as a usual regression problem: denote by $\mathbf{X}_{p}$ (respectively $\mathbf{X}_{f}$ ) the $n$ first (respectively last) rows of $\mathbf{X}$. $\hat{\mathbf{A}}^{\text {ols }}$ is naturally given by $\left(\mathbf{X}_{p}^{\top} \mathbf{X}_{p}\right)^{-1} \mathbf{X}_{p}^{\top} \mathbf{X}_{f}=$ $\mathbf{S}^{-1} \mathbf{V}=\hat{\mathbf{A}}^{\text {mle }}$. The MLE (1) is straightforwardly equivalent to the ordinary least square estimate (OLS) of $\mathbf{A}$.

Solution (1) requires a covariance matrix $\mathbf{S}$ that is invertible, which occurs when $\mathbf{S}$ is at least of rank $p$. In real situations the number of observations is often about or lower than the number of variables, thus MLE needs to be regularized. Regularization such as Moore-Penrose pseudo inversion or $\ell_{1}$-regularization can be applied on matrix $\mathbf{S}$ in order to make the inversion always achievable. A 
sharpest approach is investigated in Opgen-Rhein and Strimmer (2007), where the OLS solution is regularized by shrinking both matrices $\mathbf{S}$ and $\mathbf{V}$.

We suggest to draw inspiration from the $\ell_{1}$-penalized likelihood approach developed by Banerjee et al. (2008) in the case of i.i.d. samples of a multivariate Gaussian distribution: here, samples are no longer i.i.d yet linked through time by the VAR 1 model. Still, the sparsity can be controlled with a positive scalar $\rho$ adjoined to an $\ell_{1}$-norm penalty on $\mathbf{A}$ by solving

$$
\hat{\mathbf{A}}^{\ell_{1}}=\arg \max _{\mathbf{A}}\left\{\operatorname{Tr}\left(\mathbf{V}^{\boldsymbol{\top}} \mathbf{A}\right)-\frac{1}{2} \operatorname{Tr}\left(\mathbf{A}^{\boldsymbol{\top}} \mathbf{S} \mathbf{A}\right)-\rho\|\mathbf{A}\|_{\ell_{1}}\right\} .
$$

Since MLE and OLS are equivalent in this framework, solution to the penalizedlikelihood formulation (2) is equivalent to solving $p$ independent LAsso problems on each column of $\mathbf{A}$, which is exactly Meinshausen and Bühlmann's approach. The difference is that it does not require any post-symmetrization since there is no symmetry constraint on $\mathbf{A}$ in the present context.

\subsection{A Structured Modeling of the Network}

To attempt a better fit of data, we suggest that $\mathbf{A}$ owns an internal structure that describes classes of connectivity between the variables. Indeed, the $\ell_{1}$-norm regularization encourages a first restriction on the network's topology inferred through criteria (2), by encouraging sparsity. Yet, it is well known that by penalizing truly significant entries of $\mathbf{A}$ as much as truly zero entries a single $\ell_{1}$ penalization leads to biased estimates and a particularly strong number of false positives (Knight and Fu 2000, Zou 2006). Weighted-LAsso approaches can lower this bias by adapting penalties to prior information about where the true zero entries should be, relying on possibly data-driven as well as biological information. An existing correction is given by the Adaptive-LASso (Zou 2006, Zhou et al. 2009). Penalty coefficients are alleviated or increased using individual weights reversely proportional to an initial estimate $\mathbf{A}^{\text {init }}$.

The main purpose of this paper is to show the interest of taking into account information about the topology of the network: not only should we scale coefficients individually, but also consider the underlying organization of $\mathcal{P}$. Adaptation of weights is made by providing $\mathbf{A}$ with a well-chosen prior distribution, relying on the organization of $\mathcal{P}$. We assume that genes are spread through a partition of $\mathcal{P}$ into $\mathcal{Q}$ classes of connectivity. Both existences and weights of edges, described by the elements of $\mathbf{A}$, depend on the connectivity class each vertex belongs to. Denote by $Z_{i q}$ the indicator function that gene $i$ belongs to class $q$. Each entry $A_{i j} ; Z_{i q} Z_{j \ell}=1$ is provided with an independent prior distri-

bution $f_{i j q \ell}$. Following Ambroise et al. (2009), we choose Laplace distributions for $f_{i j q \ell}$ since it is the corresponding log-prior distribution to the $\ell_{1}$ term in the LAsso. Hence, by choosing

$$
f_{i j q \ell}(x)=\frac{1}{2 \lambda_{i j q \ell}} \exp \left\{-\frac{|x|}{\lambda_{i j q \ell}}\right\}
$$


where $\lambda_{i j q \ell}$ are scaling parameters, we expect a model whose log-likelihood will naturally make a specific $\ell_{1}$-penalization term appear.

Modeling hubs. Many configurations fit into this general model. In Ambroise et al. (2009) we focused on an affiliation model. This structure opposes intra to inter-cluster connections, assuming the former to be far more likely than the latter. In the present context, where dynamic regulatory networks are represented by directed graphs, the affiliation model unnaturally assumes symmetric probabilities for "incoming" and "outgoing" edges and should be banished. Indeed, adjacency matrices associated to directed gene regulatory networks are asymmetrical: genes belong to two completely different groups. While a group of hubs exhibits a high connection probability to all other genes, the remaining set of genes only receives edges leaving from the first class. Illustration of this phenomenon by Spellman et al. (1998)'s dataset on Saccharomyces cerevisiae is presented in Section 4. This setup can be summarized as follows:

$$
f_{i j q \ell}= \begin{cases}f_{\text {hub }}\left(\cdot ; \lambda_{\text {hub }}\right) & \text { if } q \text { is the hub class, } \\ f_{\text {leaf }}\left(\cdot ; \lambda_{\text {leaf }}\right) & \text { if } q \text { is not the hub class. }\end{cases}
$$

Note this structure only differentiate edges on the basis of their origin, whether they leave from a hub or not, whatever be their arrival points. In this type of structure built around hubs, the number of classes is fixed at 2 .

Allowing for individual prior information about $i$ and $j$, this model can be generalized to

$$
f_{i j q \ell}= \begin{cases}f_{\text {hub }}\left(\cdot ; \lambda_{i j} \lambda_{\text {hub }}\right) & \text { if } q \text { is the hub class, } \\ f_{\text {leaf }}\left(\cdot ; \lambda_{i j} \lambda_{\text {leaf }}\right) & \text { if } q \text { is not the hub class. }\end{cases}
$$

The likelihood. As the matrix $\mathbf{A}$ has been given a prior distribution, our aim is to maximize the posterior probability of $\mathbf{A}$, given the data $\mathbf{X}$. For a fixed structure $\mathbf{Z}$, this is equivalent to maximizing the joint probability

$$
\hat{\mathbf{A}}=\arg \max _{\mathbf{A}} \log \mathbb{P}(\mathbf{X}, \mathbf{A} ; \mathbf{Z}) .
$$

Now, the likelihood $\mathbb{P}(\mathbf{X}, \mathbf{A} ; \mathbf{Z})$ is straightforwardly given by

$$
\log \mathbb{P}(\mathbf{X}, \mathbf{A} ; \mathbf{Z})=\operatorname{Tr}\left(\mathbf{V}^{\top} \mathbf{A}\right)-\frac{1}{2} \operatorname{Tr}\left(\mathbf{A}^{\top} \mathbf{S} \mathbf{A}\right)-\left\|\mathbf{P}^{\mathbf{Z}} \star \mathbf{A}\right\|_{\ell_{1}}+c,
$$

where $c$ is a constant term and the $p \times p$ penalty matrix is defined by

$$
\mathbf{P}^{\mathbf{Z}}=\left(P_{i j}^{\mathbf{Z}}\right)_{i, j \in \mathcal{P}}=\sum_{q, \ell \in \mathcal{Q}} \frac{Z_{i q} Z_{j \ell}}{\lambda_{i j q \ell}} .
$$

Practically, we obtain the following penalty

$$
\mathbf{P}_{i j}^{\mathbf{Z}}=\lambda_{i j}^{-1} \cdot\left(\lambda_{\text {hub }}^{-1} Z_{i, \text { hub }}+\lambda_{\text {leaf }}^{-1} Z_{i, \text { leaf }}\right)=\rho \cdot \rho_{i j} \cdot\left(\rho_{\text {hub }} Z_{i, \text { hub }}+\rho_{\text {leaf }} Z_{i, \text { leaf }}\right),
$$


where $\rho>0$ is a common factor to $\lambda_{\text {hub }}^{-1}$ and $\lambda_{\text {leaf }}^{-1}$, which can vary so as to adapt the penalty while the ratio $\lambda_{\text {hub }}^{-1} / \lambda_{\text {leaf }}^{-1}=\rho_{\text {hub }} / \rho_{\text {leaf }}>1$ remains constant at a chosen level. Coefficient $\rho_{i j}$ can be held fixed at 1 when no individual information is taken into account or replaced by any well-chosen transformation of an initial estimate of $\mathbf{A}$ in order to provide accurate information on where true zeros might be.

\subsection{Tuning the penalty parameter}

We briefly recall here the different techniques available in the literature. Asymptotic theory of the LASSO demonstrates that a penalty parameter of order $\sqrt{n}$ guarantees both estimation consistency and selection consistency: asymptotically, estimation is unbiaised and all relevant covariates are included in the model with strictly positive probability (Knight and Fu 2000). In practice, this does not tell us which penalty to use for a fixed sample size $n$. To solve this problem, Tibshirani (1996) suggests the use of cross-validation. However it is well-known that the best penalty for prediction is not the best penalty for model selection purposes. Cross-validation is therefore unrevelant here. Optimality in terms of selection naturally draws attention towards penalties which would in some way control the false discovery rate. Closest to that goal are penalty choices which guarantee a control over the probability of connecting two nodes by a chain of edges though no such path exists in the true graph. Such penalties have been discussed in Banerjee et al. (2008), Meinshausen and Bühlmann (2006) or Ambroise et al. (2009) for instance. However, as underlined in the latter, this kind of penalty is often much too conservative to be used as anything else than an upper bound on the set of interesting penalties. Relying on the Bayesian interpretation of the LASSO, another option is to maximize the marginal probability of the data over all possible tuning parameters. A specific approximation for graphs is derived in Shimamura et al. (2007). Taking into account the fact that the number of degrees of freedom of the LASSO equals the final number of nonzero parameters (Zou et al. 2007) computations get a lot easier. Particularly, the BIC approximation of the marginal distribution as well as the AIC criterion, whose good properties for model selection are well-known, are trivial to compute. In our case, we obtain the following expressions:

$$
\begin{aligned}
\mathrm{BIC}_{\rho} & =n\left[\operatorname{Tr}\left(\mathbf{V}^{\top} \widehat{\mathbf{A}}_{\rho}\right)-\frac{1}{2} \operatorname{Tr}\left(\widehat{\mathbf{A}}_{\rho}^{\top} \mathbf{S} \widehat{\mathbf{A}}_{\rho}\right)\right]-\frac{\log (n)}{2} \mathrm{df}_{\rho}, \\
\mathrm{AIC}_{\rho} & =n\left[\operatorname{Tr}\left(\mathbf{V}^{\top} \widehat{\mathbf{A}}_{\rho}\right)-\frac{1}{2} \operatorname{Tr}\left(\widehat{\mathbf{A}}_{\rho}^{\top} \mathbf{S} \hat{\mathbf{A}}_{\rho}\right)\right]-\mathrm{df}_{\rho},
\end{aligned}
$$

where $\widehat{\mathbf{A}}_{\rho}$ denotes the estimate of $\mathbf{A}$ associated to penalty $\rho$ and $\operatorname{df}_{\rho}$ the number of nonzero entries in $\widehat{\mathbf{A}}_{\rho}$.

In practice, we observe that these last criteria present both advantages of being straightforward to compute and of providing impressively sensible results in terms of both Recall and Precision rates. We therefore adopt these criteria to select two best penalties to choose from in the remaining of the paper. 


\section{Inference Strategy}

\subsection{Structure Inference}

In many application fields, the structure can be considered as known, learned from expert knowledge. In genetic for instance, biologists can often extract the list of transcription factors from the overall set of target genes.

Otherwise, the structure, or part of it, could remain latent: we suggest a basic strategy that performs well practically for biological networks. In this context, the structure goes down to the identification of hubs. To this purpose we suggest a very intuitive path. A first matrix $\mathbf{A}_{0}$ is estimated using an adequate single LASSO penalty. We rely on AIC and BIC criteria to identify the best initial penalty. Nodes are then classified into two groups, hubs and leaves, according to the values of the $\ell_{1}$-norms of the corresponding rows in $\mathbf{A}_{0}$. In order to account for the particularly strong heterogeneity between the two groups (differences in size and dispersion), a Gaussian mixture approach is used for clustering the genes. This defines two submatrices $\mathbf{A}_{0}^{1}$ and $\mathbf{A}_{0}^{2}$ containing respectively the lines corresponding to the first and second groups. Hubs are then characterized as the class with the maximum mean absolute value of $\mathbf{A}_{0}^{k}$.

\subsection{Active-Set algorithm for Network Inference}

Once the internal structure has been recovered, inference of $\mathbf{A}$ amounts to optimizing the penalized likelihood (3) where $\mathbf{Z}$ are fixed parameters. This can be achieved by solving some $p$ independent LASSO-style problems since there is no symmetry constraint on $\mathbf{A}$ : denoting by $\mathbf{M}^{k}$ the $k^{\text {th }}$ column of a given matrix $\mathbf{M}$, we wish to solve for each column of $\mathbf{A}$ the following minimization problem

$$
\hat{\mathbf{A}}^{k}=\arg \min _{\beta} L(\beta), \text { where } L(\beta)=\frac{1}{2} \beta^{\top} \mathbf{S} \beta-\beta^{\top} \mathbf{V}^{k}+\|\Lambda \star \beta\|_{\ell_{1}},
$$

where $\Lambda=\left(\mathbf{P}^{\mathbf{Z}}\right)^{k}$ for clarity purpose.

Solving penalized problem (4) can be achieved through various algorithms. The elegant active-set approach suggested in Osborne et al. (2000) takes advantage of the sparsity of $\beta$ to solve the equivalent constrained problem: starting from $\mathbf{0}_{p}$ as an initial guess, the set of active variables $\mathcal{A}=\left\{i: \beta_{i} \neq 0\right\}$ is updated at various stages of the algorithm so as we solve linear systems with limited sizes to determine the current nonzero coefficients denoted by $\beta_{\mathcal{A}}$ herein. The algorithm stops once the optimality conditions derived from the classical Karush-Kuhn-Tucker conditions are satisfied. In the next paragraph, we detail an adaptation to the present context of the Osborne et al., initially developed for the LASSO for linear regression.

The objective function $L$ in (4) is convex, yet not differentiable everywhere due to the $\ell_{1}$-norm: from convex analysis, $\beta$ is solution to (4) iif $\mathbf{0}_{p}$ belongs to 
the subdifferential of $L$, which mainly forms the optimality conditions of the problem. Here, the subdifferential is given by

$$
\partial_{\beta} L(\beta)=\mathbf{S} \beta-\mathbf{V}^{k}+\Lambda \star \boldsymbol{\theta},
$$

where $\boldsymbol{\theta} \in \operatorname{sign}(\beta)$, that is, $\theta_{i}=\operatorname{sign}\left(\beta_{i}\right)$ if $i \in \mathcal{A}$, and $\theta_{i} \in[-1,1]$ if $i \in \overline{\mathcal{A}}$.

Starting from $\beta=\mathbf{0}_{p}$, we select the component $\ell$ of $\beta$ whose subgradient absolute value is maximal: as a matter of fact, a subgradient highly different from zero induces high violation of the optimality conditions. Such a choice will guarantee a large reduction of the objective function $L$ during the optimization procedure. Thus, this component is added to the active set $\mathcal{A}=\mathcal{A} \cup\{\ell\}$.

Then, optimization is only performed on nonzero coefficients $\beta_{\mathcal{A}}$ whose cardinal is small since the solution is likely to be sparse. This is done by minimizing $L\left(\beta_{\mathcal{A}}\right)$, which reduces to a classical optimization problem because the subdifferential turns to an usual gradient $\nabla_{\beta} L$ on the active set $\mathcal{A}$.

While optimizing, the next update $\beta_{\mathcal{A}}^{+}=\beta_{\mathcal{A}}+\mathbf{h}$ is obtained by solving $\nabla_{\mathbf{h}} L\left(\beta_{\mathcal{A}}+\mathbf{h}\right)=\mathbf{0}_{|\mathcal{A}|}$, which leads to the following descent direction

$$
\mathbf{h}=-\beta_{\mathcal{A}}+\mathbf{S}_{\mathcal{A}, \mathcal{A}}^{-1}\left(\mathbf{V}_{\mathcal{A}}^{k}-\Lambda_{\mathcal{A}} \star \operatorname{sign}\left(\beta_{\mathcal{A}}+\mathbf{h}\right)\right) .
$$

However $\operatorname{sign}\left(\beta_{\mathcal{A}}+\mathbf{h}\right)$ cannot be known while computing $\mathbf{h}$ and is consequently approximated by the current sign of $\beta_{\mathcal{A}}$ equal to $\boldsymbol{\theta}_{\mathcal{A}}$ :

$$
\mathbf{h} \approx-\beta_{\mathcal{A}}+\mathbf{S}_{\mathcal{A}, \mathcal{A}}^{-1}\left(\mathbf{V}_{\mathcal{A}}^{k}-\Lambda_{\mathcal{A}} \star \boldsymbol{\theta}_{\mathcal{A}}\right) .
$$

Due to this approximation, we check for sign-consistency between the candidate update $\beta_{\mathcal{A}}+\mathbf{h}$ and $\boldsymbol{\theta}_{\mathcal{A}}$. In case of inconsistency, the descent direction is reduced so as $\beta_{\mathcal{A}}+\gamma \mathbf{h}$ is sign consistent with $\boldsymbol{\theta}_{\mathcal{A}}$. This ends the optimization part of the algorithm.

Then, the active set $\mathcal{A}$ is updated since some $\beta_{i}$ could have been set to zero during the optimization procedure: this is done by looking for vanished $\beta_{i}$ s, verifying $\partial_{\beta} L\left(\beta_{i}\right)=0$. Finally, optimality conditions are tested: if the maximal $\ell$ of the subdifferential corresponding to an unactivated component of $\beta$ is zero, we have found a solution; otherwise, the active set is updated by adding $\ell$ to $\mathcal{A}$, since it induces the highest reduction of $L$.

These three steps - optimization, deactivation and optimality testing - are repeated until a solution has been found, which is guaranteed (see Osborne et al. 2000). The full algorithm is detailed below. Note that it can either start from $\beta^{0}=\mathbf{0}_{p}$ or from a solution obtain from a more penalized problem with larger vector of penalties $\Lambda$, that speeds up the computation, hence having a behavior that is similar to the homotopy/LARS algorithm (Efron et al. 2004).

The full matrix $\mathbf{A}$ is directly recovered by binding column-wisely the solutions to the $p$ LASsO-style problems.

Remark. With this method, the sparsity constraint only applies to each column of $\mathbf{A}$. This constraint implies that if we use $n+1$ time points, $\mathbf{S}$ is of rank $n$ and thus no more than $n$ connections can be activated by the LAsso at most in each column (assuming the penalty is low enough to accept the activation of all 


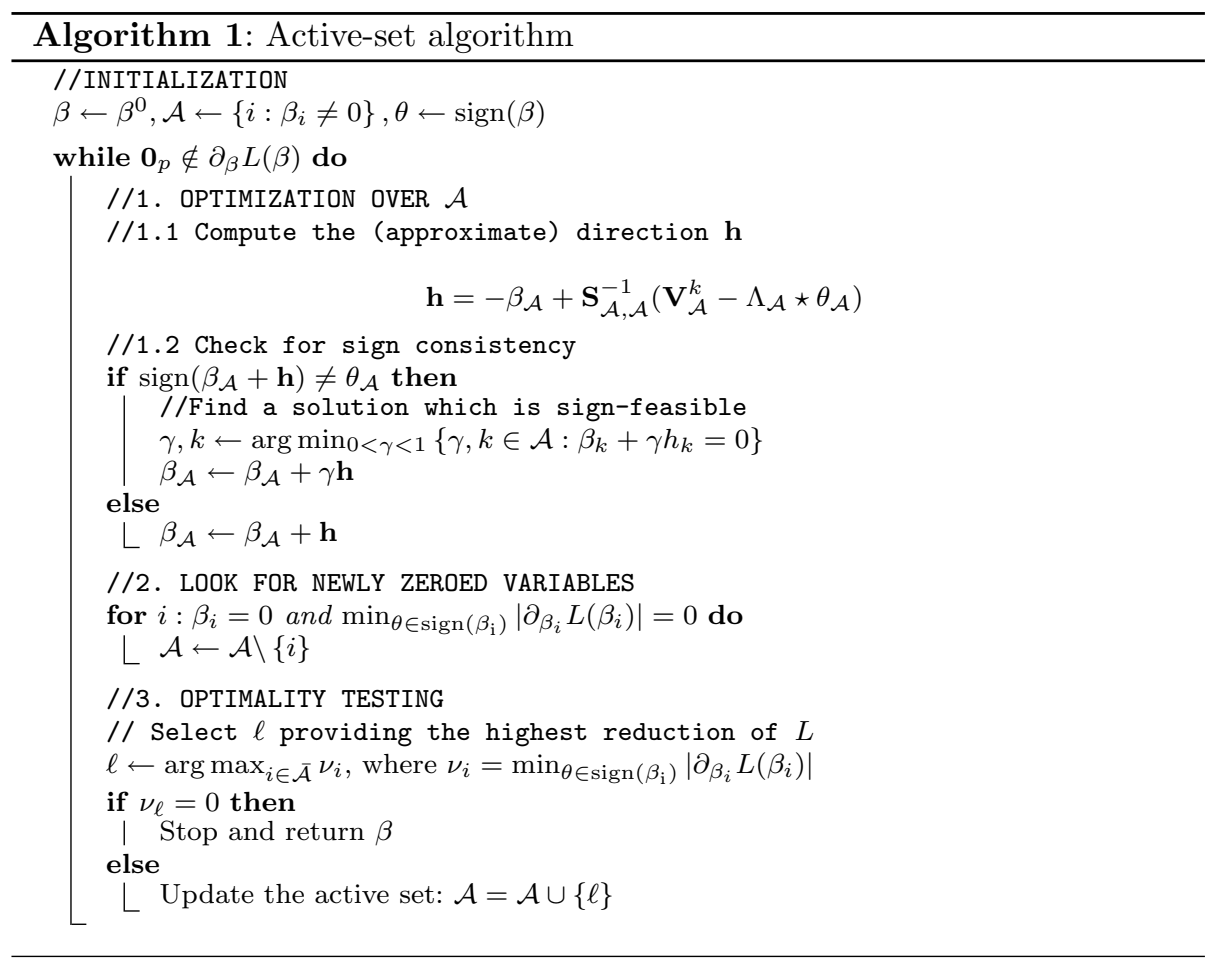


possible edges). Consequently, the sparsity constraint only applies to incoming edges and not to outgoing ones. In that sense, sparsity assumptions implied by $\ell_{1}$ penalization only assume that each node is regulated by a small set of nodes and do not contradict the existence of hubs regulating a huge set of nodes.

\section{Experiments and Discussion}

In this section we apply our algorithm to both synthetic and real data. Comparison is made first within the family of the weighted-LAsso. We observe the performances of the LASSO when associated with a single LASSO penalty or an adaptive penalty. For the adaptive-LASSO, a single LASSO penalty is used as initial estimator. We then try two different hub penalties: one relying only on the known hub structure and a last one inferring the hub structure from the initial LASso estimator. We denote these estimators by Lasso, Adaptive, KnwCl, and $\mathrm{InfCl}$ respectively. Corresponding penalties can be summarized as follows:

$$
\begin{aligned}
P_{i j}^{\mathrm{Lasso}} & \propto 1 \\
P_{i j}^{\text {Adaptive }} & \propto\left(\frac{1}{\hat{A}_{i j}^{\text {init }}} \vee 1\right) \\
P_{i j}^{\mathrm{KnwCl}} & \propto\left(\rho_{\mathrm{hub}} Z_{i, \text { hub }}+\rho_{\text {leaf }} Z_{i, \text { leaf }}\right) \\
P_{i j}^{\mathrm{InfCl}} & \propto\left(\rho_{\text {hub }} \hat{Z}_{i, \text { hub }}+\rho_{\text {leaf }} \hat{Z}_{i, \text { leaf }}\right),
\end{aligned}
$$

where $x \vee y=\max \{x, y\}$ and $\hat{Z}$ denotes the inferred classification. In the remainder of this section, we fix the ratio $\rho_{\text {leaf }} / \rho_{\text {hub }}=2$, thus penalizing twice as much nodes labeled as leaves as nodes labeled as hubs. Note also that we choose to maintain the modification of adaptive weights adopted in Zhou et al. (2009) and prevent the alleviation of penalty parameters. This trick ensures that the adaptive LASSO will select a subnetwork from the network inferred by the initial LAsso estimate. No edge can be included if it was already excluded by the LASSO. In this way, the adaptive LASSO guarantees a decrease in false positives.

Apart from our family of weighted-LAsso proposals, comparison will be made with state-of-the art network inference methods in a VAR1 setting: the Shrinkage method suggested by Opgen-Rhein and Strimmer (2007), the Recursive Elastic Net method (Renet-VAR) developed by Shimamura et al. (2009) and the method based on dynamic Bayesian networks proposed by Lèbre (2009) and available in $R$ within the G1DBN package.

Here, the interest of the inference lies in the recovery of the true edges, in other words of whether the entries of $\mathbf{A}$ are correctly identified as nonzero. Our estimators are mainly used for discriminating nonzero entries from others. Quantities such as True Positives (TP), False Positives (FP), True Negatives (TN) and False Negatives (FN) summarize the performances of these classifiers. Precision $\mathrm{TP} /(\mathrm{TP}+\mathrm{FP})$ is the ratio of the number of true nonzero elements to the total number of nonzero elements in the estimated matrix $\hat{\mathbf{A}}$. Recall $\mathrm{TP} /(\mathrm{TP}+\mathrm{FN})$ denotes the proportion of nonzero elements in $\mathbf{A}$ which were 
correctly recovered as nonzero in the estimation. Fallout $\mathrm{FP} /(\mathrm{FP}+\mathrm{TN})$ gives on the contrary the proportion of zero elements in $\mathbf{A}$ which were falsely declared as nonzero in the estimation. In statistical terms, the Recall (or Hit Rate) would be the empirical equivalent of the power of our classification method considered as a test, while the Fallout (or False Alarm Rate) would correspond to the first type $\alpha$ error. Note that, in the context of sparse network inference, the number of total positives is small compared to the number of total negatives. Thus, small variations of FP and TP will induce small variations in Fallout and large variations in Recall. Hence, comparison between Precision and Recall is generally more relevant than Fallout / Recall comparison in the present sparse context. This is why we will generally choose to omit Fallout rates when we need to alleviate the presentation of results.

These rates are easily obtained for the LAsso based methods since they automatically produce null coefficients. By increasing the penalty parameter we obtain sparser and sparser graphs. We start from a large enough penalty to constrain all coefficients of $\hat{\mathbf{A}}$ to 0 and decrease the penalty until we include as many variables as allowed by the ratio $n / p$. We then select the best penalty from this list as the one maximizing either the BIC or the AIC criterion.

Like the LASSO, Renet-VAR directly implements variable selection and penalty choice is included in the algorithm. Concerning G1DBN, we follow the author's advice to tune the parameters of the test procedure as described in the additional material of Lèbre (2009). When applying the Shrinkage method developped by Opgen-Rhein and Strimmer (2007), a supplementary step is required to transform continuous results into a binary solution. We follow Opgen-Rhein and Strimmer's advice and rely on local false discovery rates. This provides each edge with an existence probability conditional on the corresponding entry in $\hat{\mathbf{A}}$. We declare as inferred edge any edge with posterior probability exceeding the threshold of $80 \%$ as the authors do.

\subsection{Simulated Data}

Simulation settings. To assess the performances of our approach, we apply the previous model to a very favorable setup, where existing models already perform quite well. We then decrease the ratio $n / p$ in order to observe the response of each method to this increasing lack of information. On top of that, we consider graphs of different sizes: small graphs of 20 nodes, larger graphs of 100 nodes and a setup with 800 nodes. For smaller graphs, we consider three different amounts of observations: 10, 20 and 40. For medium sized graphs, we also consider the cases $n=p / 2$ and $n=p$ but omit the case $n=2 p$ as unrealistic. The setup $p=800, n=20$ is meant to mimic Spellman et al.'s dataset.

Simulation of the VAR1 process is based upon the simulation strategy used by Opgen-Rhein and Strimmer in order to ease the comparisons, but introduces a structure based on hubs in order to better reflect the structure we could expect from a real data set. A graph is first simulated, with fixed numbers of 
nodes and edges. Like Opgen-Rhein and Strimmer we simulate sparse graphs, with $K=2 p$ edges. Nodes are split into two groups according to a multinomial distribution with probabilities $(0.1,0.9)$, leading to $10 \%$ of hubs in average. Edges are then positioned in the graph according to a multinomial distribution, with $85 \%$ of edges from hubs to leafs, and the remaining set within hubs. Exception is made for the very large graph, for which we base the number of edges and their distribution on Spellman et al.'s data. The matrix $\mathbf{A}$ is synthesized on the basis of this graph: we attribute a random partial correlation value uniformly distributed on $[-1,-0.2] \cup[0.2,1]$ to all nonzero coefficients (corresponding to edges in the graph).

From this matrix, a VAR1 observation is generated, using a centered Gaussian starting value and a centered Gaussian noise, both with variance $\sigma^{2}=0.1$. For computing time reasons, this is repeated 500 times for the small graphs, 200 times for medium sized graphs and 100 times for the large graph. Results are averaged over all samples.

To gain a better insight into the difficulty of these synthesized data set for a LASSO estimator, we checked whether the irrepresentability condition (Zhao and Yu 2006, Meinshausen and Yu 2008) was validated in all these very simple simulations. First, note that the graphical context requires the irrepresentability condition to be validated for each of the $p$ genes at the same time, which makes it much more difficult to hold than in the simple regression context where it is an already strong hypothesis. In our context, since we solve $p$ independent LASSO problems, we can check the validity of the hypothesis in each of these individual problems. For each gene, the irrepresentability condition is tested using the true sign pattern extracted from the corresponding column of the true adjacency matrix. Thus the sets of relevant and irrelevant covariates are allowed to vary from one problem to another. Simulating 100 samples of each simulation setting, we observed that even in a favorable setup with twice as many observations as variables ( $p=20$ genes) the irrepresentability condition fails for $30 \%$ of genes in average. With $p=20$ genes and only $n=10$ observations this assumption fails in average for $51 \%$ of the genes. In other words, for around half of the genes we cannot expect the LASSO to recover the exact sign pattern. See Table 1 for details. Admittedly, the irrepresentability condition is a really strong assumption, necessary and sufficient for exact sign recovery, that is to say not only the exact neighborhoods (no false positives, no false negatives) but also the exact signs of the correlations. Yet since the simulated values are quite well separated between true zeros and true nonzeros we would have expected that this hypothesis would have been much more validated. Information about the validity of the restricted eigen-value assumptions (Bickel et al. 2009) would be greatly appreciated to compensate for such pessimistic results, but these are computationally intractable. Adaptation of Juditsky and Nemirovsky (2008)'s results to the present context could be of great benefit.

Discussion of simulation results. Results are presented in Figure 1 under the form of Barcharts. Figure 2 illustrates the case where $p=100$ by giving 


\begin{tabular}{c|cc}
$n / p \backslash^{p}$ & 20 & 100 \\
\hline 2 & $0.30(0.23)$ & - \\
1 & $0.41(0.23)$ & $0.37(0.15)$ \\
$1 / 2$ & $0.51(0.18)$ & $0.42(0.12)$ \\
& TABLE 1
\end{tabular}

Average proportion of genes for which the irrepresentability condition does not hold and standard error in each simulation setting.

boxplots for the distributions of Precision, Recall and Fallout.

Compared methods differ with the type of setting. First of all, since the Shrinkage method (particularly the local false discovery rate step) relies on the hypothesis that $p$ is large, we do not consider it fair to apply it to the small network setting. Reversely, for computing time reasons we decided to restrict the application of $G 1 D B N$ to the graphs of size $p=20$.

Penalties for the LASSO based methods were chosen on the basis of either the BIC or AIC criterion. Although theory states that the BIC ought to outperform the AIC in terms of model selection (Zou et al. 2007), we observed that in practice the BIC criterion might be too conservative when $n$ is small compared to $p$. In that situation, it might be interesting to favor the less stringent AIC criterion which will induce a higher recall rate for not such a large loss in precision. Note that the penalty choice based on the AIC or the BIC can lead to choose the null model as best model. In that case, Precision cannot be defined. We thus show the results for precision over all simulations where at least one variable was included.

The first point worth noting in Figure 1 is that in all settings the LASSO is outperformed by weighted-LASSO methods and others. This quick check confirms the interest of compensating for the bias induced by $\ell_{1}$ regularization on large coefficients. It is also possible that what we observe about the validity of the irrepresentability condition jeopardizes the performances of the singlepenalty LASSO. In line with Table 1, the LASSO performs particularly badly when the ratio $n / p$ is not favorable, with recall and precision rates under $20 \%$ when $p=20, n=10$. It even performs so poorly that it deprecates the inference based on adaptive weights. A priori information on where the true zeros might compensate for this apparent lack of "neighborhood stability", using Meinshausen and Bühlmann's vocabulary, and explain why the $\mathrm{KnwCl}$ penalty is far more accurate (precision of $84 \%$ in average for a recall of nearly $50 \%$ in average for the same simulation setting $p=20, n=10$ ).

As expected, in all settings (except when $n$ is really too small compared to $p$ ) the Adaptive penalty improves the precision but at the price of a smaller recall rate. On the contrary, the inferred classification $\mathrm{InfCl}$ allows to improve the precision without undermining the recall rate. However, both methods are highly dependent on the initial LASSO estimate. Therefore, the gain in precision resulting from such methods decreases with the $n / p$ ratio.

Benefitting from a certain amount of supplementary information, the $\mathrm{KnwCl}$ penalty leads to a clear increase in both precision and recall. Particularly when little information is available in terms of number of observations, taking a priori 
information about which genes are potential regulators and which are not into account improves the results dramatically. This is true when compared to all LAsso based methods but generalizes to Shrinkage, Renet-VAR and G1DBN. Admittedly, Renet-VAR leads to higher precision values with medium sized graphs, but it is compensated by smaller recall rates.

Table 1 shows naturally that we cannot expect too much from very extreme settings ( $p=800, n=20$, that is, the Spellman et al.'s settings). Average Recall rate is less than $20 \%$ for all methods except the $\mathrm{KnwCl}$ penalty. In this case, knowledge of potential hubs allows the recall rate to almost double in average while increasing the precision. Note however that even with this supplementary information precision rates never exceed $50 \%$.

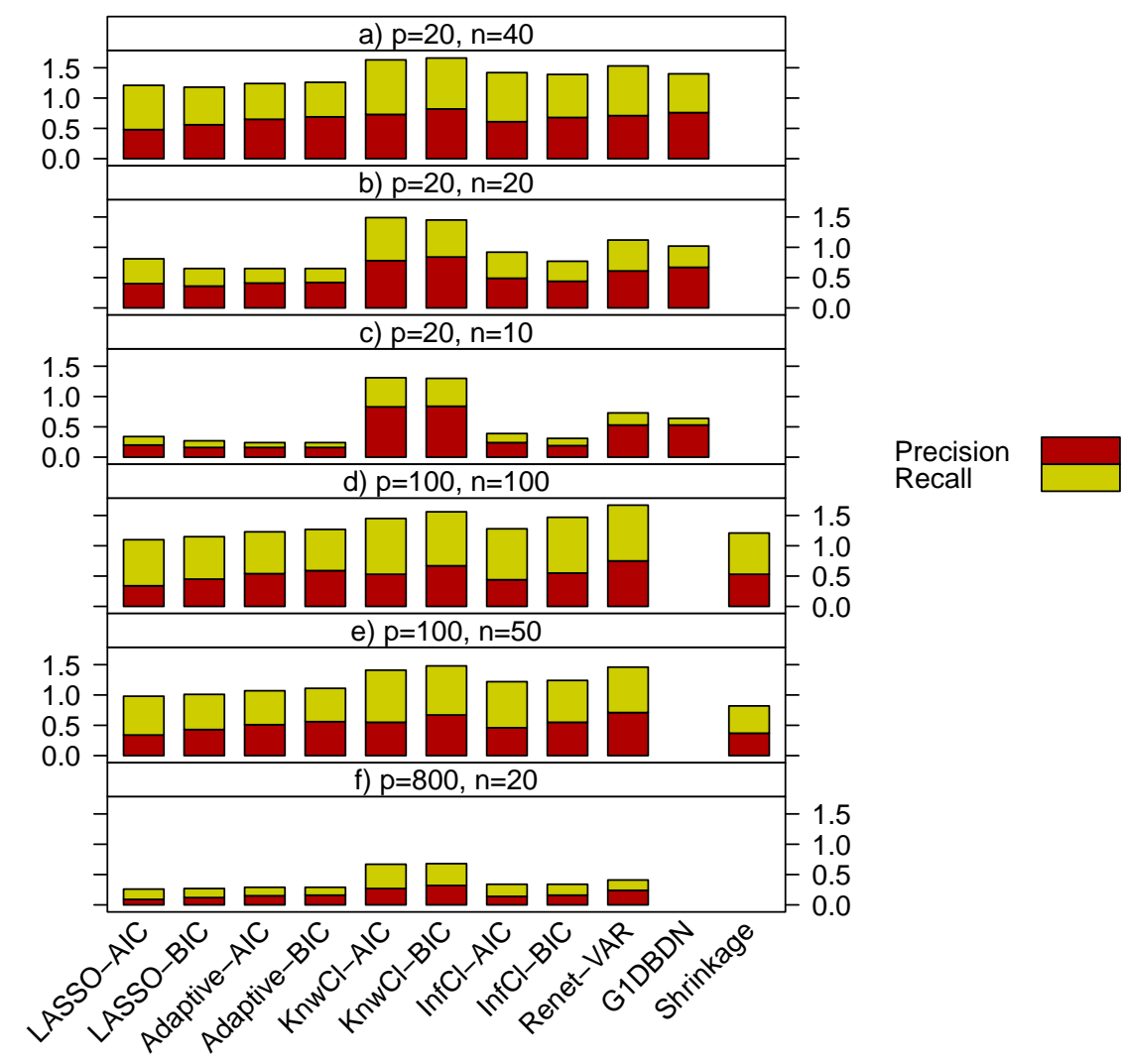

Fig 1. Bar charts of Precision and Recall rates for each method and simulation setting, averaged over all simulation samples.

To finish with, we would like to lay the emphasis on computing times. For 


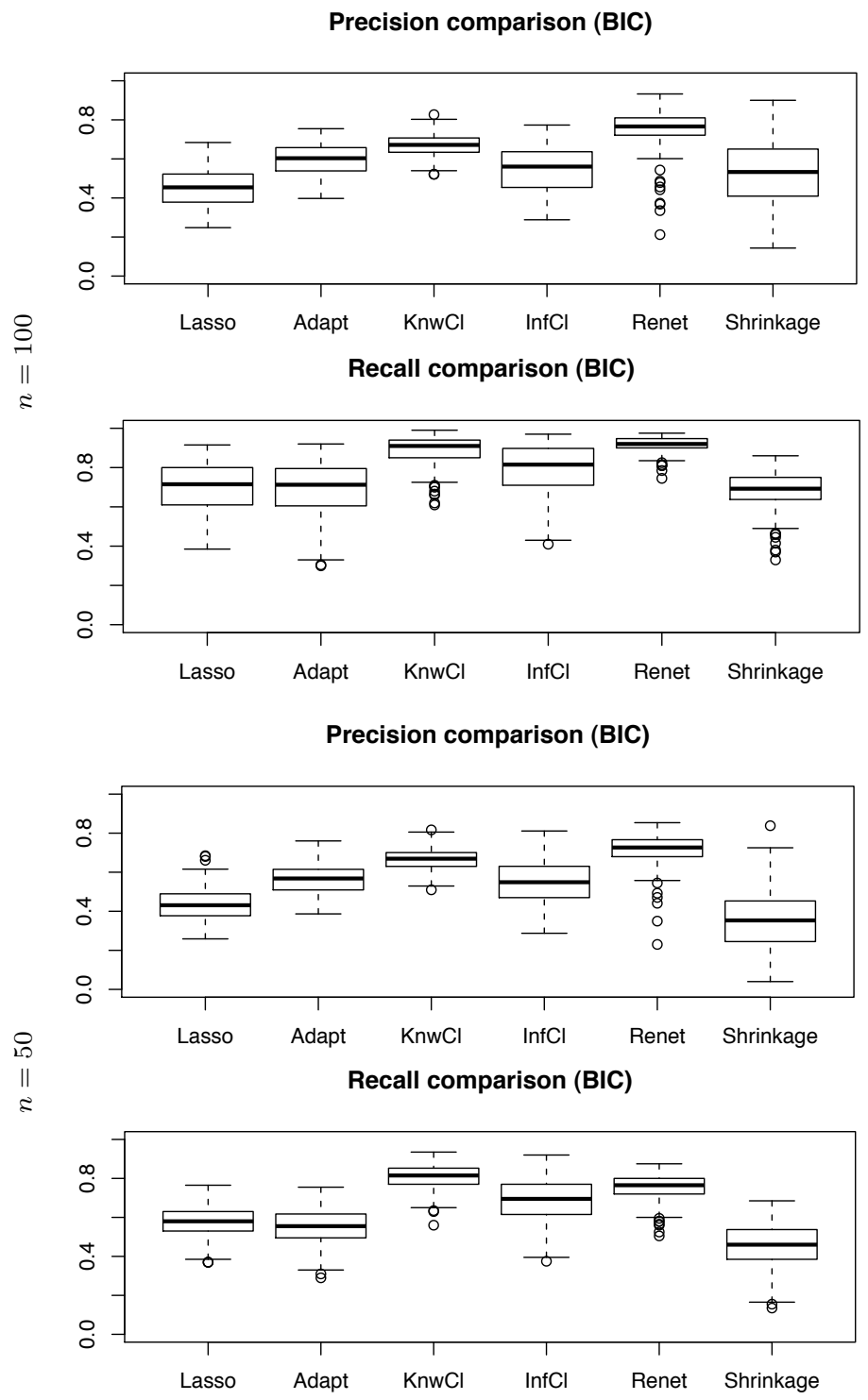

Fig 2. Boxplots of Precision, Recall and Fallout statistics for all methods except Shrinkage in a setup $p=100$, for 200 simulated data sets. Best Lasso penalties chosen on the basis of the BIC criterion.

this we let the number of nodes range from 5 to 185 and fixed the number of observations at half the maximum number of nodes, i.e. $n=92$. This leads to a ratio $n / p$ ranging from 0.05 to 2 . Computing times for the weighted LASSO with inference of the classification InfCl and selection of the best penalty, Renet$V A R$ and $G 1 D B N$ are presented in the $\log -\log$ scale in Figure 3. We can see 
that running times for Renet-VAR and G1DBN can become a handicap as soon as $p$ gets large while computing times for $\mathrm{InfCl}$ rarely exceed 2 minutes.

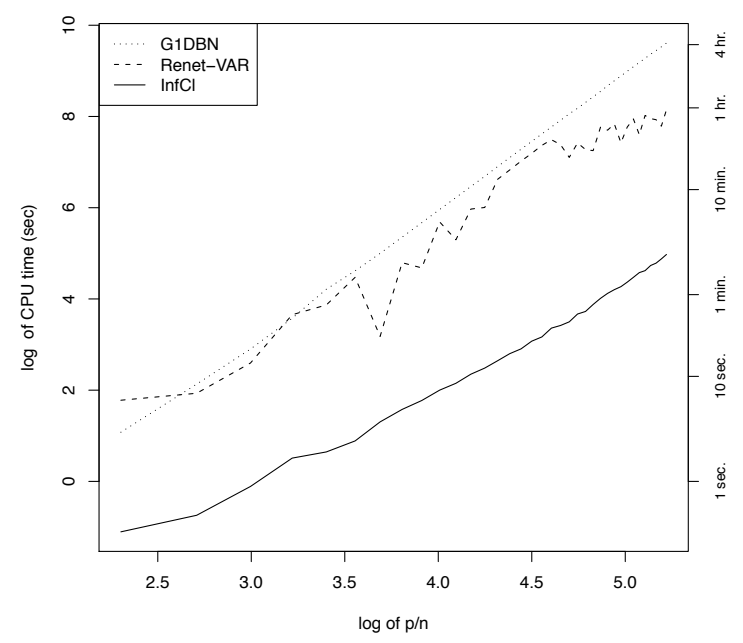

FIG 3. Computing times on the log-log scale for Renet-VAR, G1DBN and InfCl (including inference of classes). Intel Dual Core $3.40 \mathrm{GHz}$ processor.

\subsection{Yeast Data}

We confronted our model to time measurements of Saccharomyces cerevisiae gene expression data collected by Spellman et al. (1998). We focus on the subset of genes they identified as periodic, i.e. genes whose transcription levels over time show evidence that they are cell-cycle regulated.

Remarks on the data set. This dataset is one of the first microarray experiments. It is thus doomed to be rather noisy, contrary to the simulated data sets. Besides, we had to face the problem of missing values, which appeared on some of the most important genes. We imputed them as the mean of the two closer known observations in time for the gene considered, before and after the time point of interest.

On top of its noisiness, Spellman et al.'s data set is particularly hard to tackle from a statistical view point. Information is provided on 786 genes for only 18 time points. This implies that using our algorithm we cannot activate more than $17 * 786=13362$ edges out of $789 * 786=617796$ possible ones, that is to say $2.2 \%$.

However, we can rely on experimental conclusions on yeast gene regulation networks to collect target information about the true edges of the graph. We compare our results to the adjacency matrix provided by the Yeastract database (www.yeastract.com). We retain information on documented direct relation- 
ships, that is to say direct regulations confirmed by published experimental results.

Note however that this theoretical benchmark is biased in two ways. First, some true edges might be missing because all regulations might not have been confirmed by experiments yet. Second, this graph gathers all reported regulations, whatever the conditions of the experiment. Some might not actually happen during the precise experiment we consider. We can suppose the effect of the first bias to be low in a model organism such as Saccharomyces cerevisiae. The effect of the second bias is much more likely however, since measurements are all made while cells are at the beginning of their growth, growing until ready for DNA synthesis. We cannot expect the whole range of possible regulations to happen in such a small portion of the cell cycle.

This dataset illustrates quite well the biological properties our model is based upon. First, documented information reveals the existence of 1385 true edges (among more than 600000 possible ones in theory). The theoretical graph is thus extremely sparse. Secondly, the hub structure is quite clear: edges leave from only 26 out of 786 genes. Hence knowledge of the hubs provides crucial information on the position of edges. This phenomenon also clearly appears on Figure 4. Incoming degrees never exceed 20 but only 1 is null. On the contrary, outgoing degrees are null for the vast majority of genes. Significant degrees appear as outliers in this distribution, reaching up to 150 for some of them.

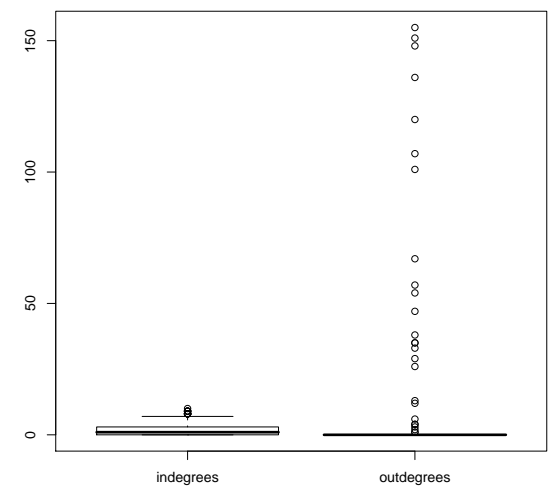

FIG 4. Boxplots of incoming and outgoing degrees in Yeast theoretical adjacency matrix

Discussion of the results. The setting is much harder than in the first simulated data sets, with a ratio $n / p=2.3 \%$ as well as harder than the last simulated dataset with less separated correlations between existing and non existing edges. Results presented in Table 2 show quite well the difficulty all methods encounter in front of this data set. Results for the Shrinkage approach 
are not shown because the local false discovery rate step included in this method was heavily flawed by the lack of separability between edges and non edges. Except for the $\mathrm{KnwCl}$ penalty, all LAsso based estimators are reduced to the null model. Both the BIC and AIC criteria do not find the increase in likelihood large enough to compensate for the complexity of any model with at least one edge. Performances of the $\mathrm{KnwCl}$ penalty and Renet-VAR remain lower than what we could expect from simulated results.

\begin{tabular}{lcccccc}
\cline { 1 - 5 } Models & & Lasso & Adaptive & KnownCl & InferCl & Renet \\
\cline { 1 - 5 } \cline { 3 - 7 } Precision & & - & - & 0.082 & - & 0.004 \\
Recall & & 0 & 0 & 0.068 & 0 & 0.003 \\
Fallout & 0 & 0 & 0.002 & 0 & 0.002 \\
\hline
\end{tabular}

Precision, Recall and Fallout performances for all Lasso based methods and Renet-VAR on Spellman et al.'s data set. Best Lasso penalties chosen on the basis of the BIC criterion.

Many reasons for such bad perfomances could be thought of. We already mentionned the noisiness of the data, which quite hardly differentiated the edges from non edges. Second, homogeneity of the VAR(1) model might be too strong an assumption. Last but not least, when looking more closely at how data were collected we noticed that measurements were made every 7 minutes, which might be long enough for dependencies to vanish. Also, since we measure values related to the cell cycle, measurements were necessarily made on different cells each time, thus measuring the expression levels on different individuals at each time point. In brief, this apparently longitudinal data set might share more common points with i.i.d. models than with VAR1 processes.

\subsection{E. coli S.O.S. DNA repair network}

In this section we quit the high dimensional setup and compare the performances of all methods in a much easier framework. We focus on a sub-network from $E$. Coli S.O.S. DNA repair network analyzed by Ronen et al. ${ }^{1}$. Data provide information on the main 8 genes of the S.O.S. network (uvrD, lexA, umuD, rec $A$, uvr $A$, uvr $Y, \operatorname{ruv} A$, polB $)$ across 50 time points. Measurements rely on precise expression kinetics which allow Ronen et al. to monitor mRNA expression levels every 6 min after exposition of the DNA to UV light at time 0 . We will not dwell on the measurement technology here (see Ronen et al. (2002) for details). Note however that the authors do not measure the actual mRNA quantity present in the cell at time $t$ but the instant promoter activity of each gene. Equivalence between the two measurements is guaranteed if the instant quantity of mRNA in the cell roughly equals its production rate, that is to say if there is no accumulation of mRNA in the cell. Under this assumption, Ronen et al. 's data can be used as any microarray dataset.

E. coli S.O.S. DNA repair network provides a precise benchmark: specific regulatory interactions in response to DNA damage have been characterized. In

\footnotetext{
${ }^{1}$ data downloadable on Uri Alon's homepage, http://www . weizmann.ac.il/mcb/UriAlon/
} 
other words, we can rely on a theoretical regulatory network which represents the main direct transcriptory regulations actually taking place during the experiment. According to the regularly updated EcoCyc database, lexA is the only regulator in this subnetwork, regulating all genes including itself. Concretly, the protein $\operatorname{LexA}$ is at the core of the regulation network, usually binding sites in the promoter regions of S.O.S. genes to repress their expression. As soon as RecA senses DNA damage (by binding to single-stranded DNA), it becomes activated and induces LexA autocleavage. The decrease in LexA concentration alleviates the repression of S.O.S. genes. When damage is repaired, the level of activated RecA drops, LexA accumulates and represses again all S.O.S. genes.

Detailed results are presented in Figure 5. We can see that performances differ a lot from one experiment to another. Particularly, experiments 1 and 4 lead to significantly poor results although nothing should a priori distinguish them from 2 or 3 ( 1 and 2 , respectively 3 and 4 , share the same U.V. exposure).

As on simulated data, the LAsso leads to poor results. G1DBN shows similarly poor performances here. Quite surprisingly, Renet-VAR does not perform as well as we could have expected from simulations. It reaches $50 \%$ of recall at the expense of very low precision rates. Adaptive penalty improves more the quality of the estimation than in the simulation studies. Now they increase the precision of the LASSO without really undermining the recall rate. Inference of the classification outperforms these, with higher recall and precision rates. This is quite interesting since except in experiments 1 and 4 where the LASSO provide almost no information, inference of the classes seems quite good although the initial LASSO still shows mediocre results. To finish with, the $\mathrm{KnwCl}$ penalty benefits quite well here from its extra information since it outperforms all other methods and manages to reach honest results even in datasets 1 and 4 which disturbed all other methods.

Inferred graphs on experiment 2 are shown in Figure 6. The regulatory activity of lexA is more or less recovered by all methods. What is interesting is that a common structure recurently shows up among false positives: regulations due to uvr $A$. This regulation pattern is particularly what dominates experiment 4 and leads to so poor results. Strangely, we could not find any mention of this regulatory activity in the literature. Either there is a need for further biological research on this gene or there is an undirect regulation blurring the results. Another unknown regulation dominates all inferred graphs: regulation of uvr $Y$ by polB. It is all the more interesting as it survives the bad a priori that the $\mathrm{KnwCl}$ penalty holds against it. Further biological investigation could want to look at this couple of genes more closely.

In this respect, we could note that the regulatory effect of activated $\operatorname{Rec} A$ on LexA does not appear on these graphs, which we could see as a good point since this is a post-transcriptional regulation. We would also like to lay the emphasis on the fact that we here check selection consistency of all the methods but not their sign consistency. We only check whether we identify the right edges and not the activation/inhibition processes associated to them. Looking more closely at the estimated matrices, we can see that the (shrunk) correlations estimated between lexA and the remaining genes are all positive and not negative 


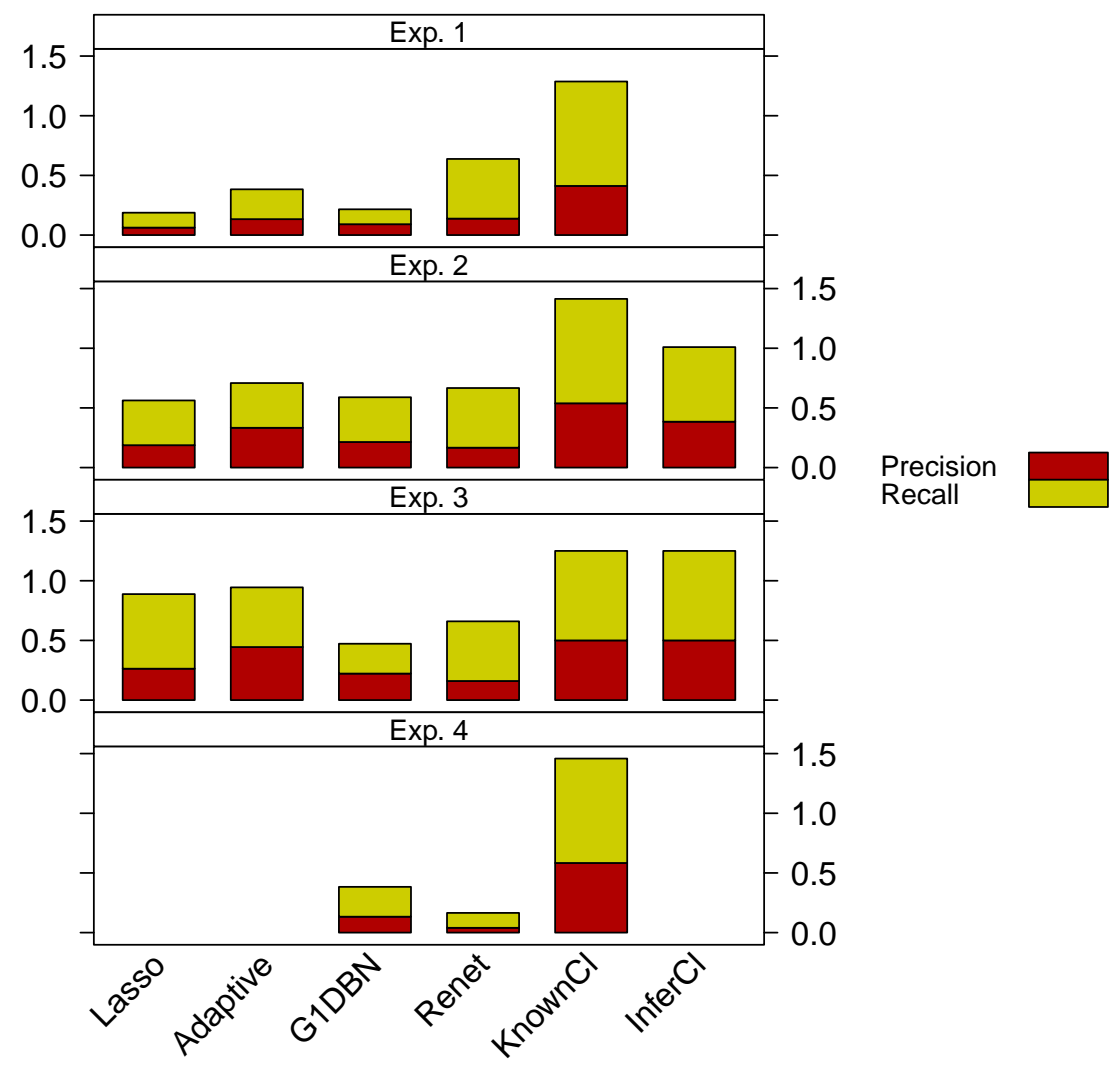

FIG 5. Summary of Precision, Recall and Fallout (respectively Prec., Rec. and Fall.) values for each method and experiment

as the literature would tell. This would not be a flaw in all methods but a direct result of the limitations of transcriptomic data. Indeed, we only observe mRNA production rates. As a consequence, we cannot spot the decrease in concentration of protein LexA and only observe that the expression of all genes suddenly increases, lexA included.

\section{Conclusion}

This paper proposes a weighted-LASSO algorithm designed to tackle time varying gene expression data taking into account an underlying structure. We observe that in a perfect VAR1 setting, taking time dependencies into account leads to dramatically improved results for graph inference. In this particular framework, 

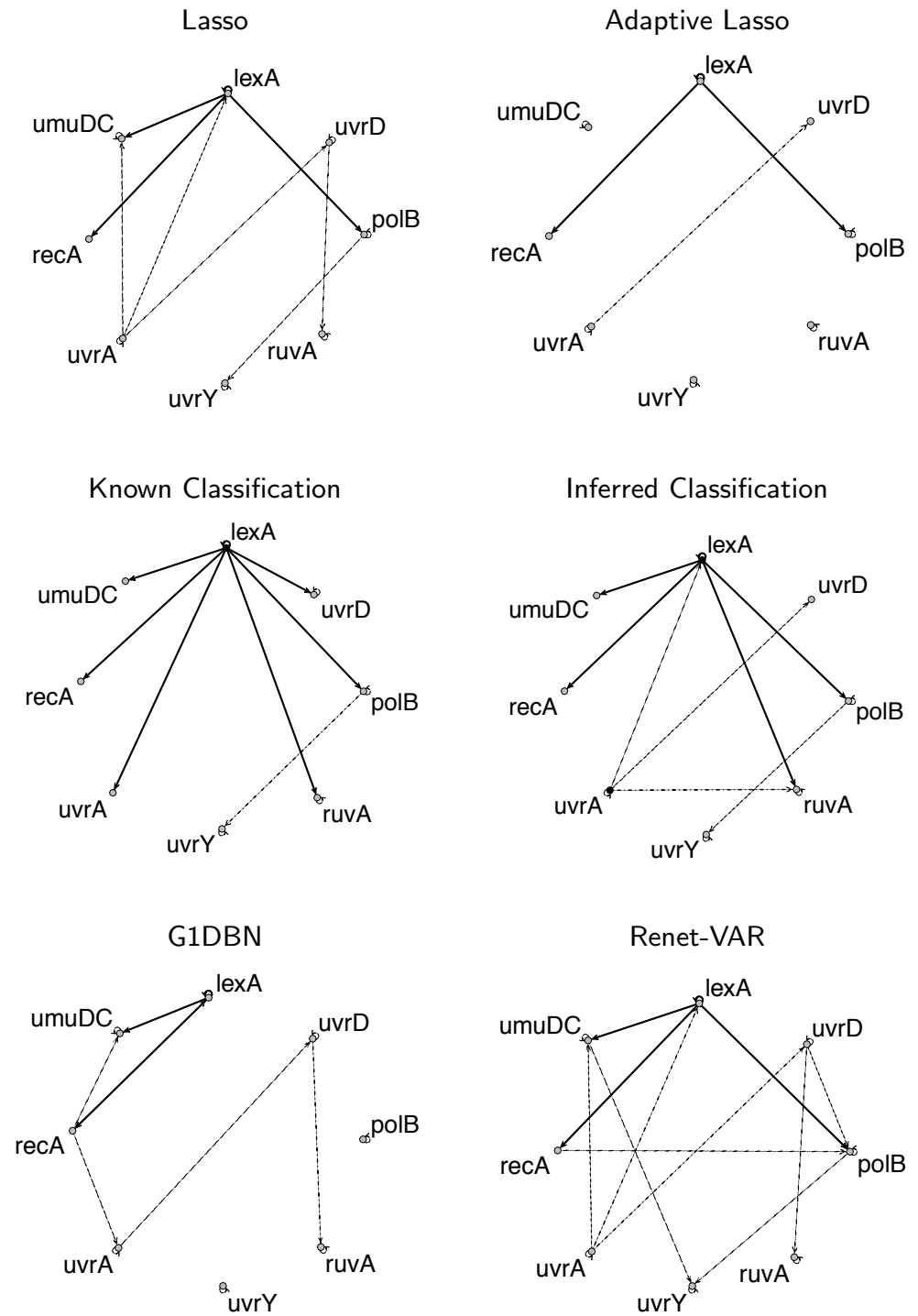

FIG 6. Graphs inferred by the different methods on experiment 2 data. LASSO penalties are chosen so as to maximize the BIC criterion. True positives are drawn in black while false positives are shown in dashed gray.

the proposed approach outperforms similar methods. Even when regulators and regulatees cannot a priori been distinguished through analysis of the literature, inference of the classification improves a lot the performances of the LASsO. It therefore seems good to advice that, whenever available, knowledge about potential transcription factors should be taken into account and that basic knowledge 
on the topology of biological networks should not be omitted in the modeling process. We also want to emphasize the fact that this method reaches great results on networks of reasonable size for always reasonnable computing times.

\section{References}

C. Ambroise, J. Chiquet, and C. Matias. Inferring sparse Gaussian graphical models with latent structure. Electronic Journal of Statistics, 3:205-238, 2009.

O. Banerjee, L. El Ghaoui, and A. d'Aspremont. Model selection through sparse maximum likelihood estimation for multivariate Gaussian or binary data. $J$. Mach. Learn. Res., 9:485-516, 2008.

P. Bickel, Y. Ritov, and A. Tsybakov. Simultaneous analysis of lasso and dantzig selector. Annals of Statistics, 37:1705-1732, 2009.

R. Castelo and A. Roverato. A robust procedure for Gaussian graphical model search from microarray data with $p$ larger than $n$. J. Mach. Learn. Res., 7: 2621-2650, 2006.

J. Chiquet, A. Smith, G. Grasseau, C. Matias, and C. Ambroise. Simone: Statistical inference for modular networks. Bioinformatics, 25(3):417-418, 2009.

A.P. Dempster. Covariance selection. Biometrics, Special Multivariate Issue, 28:157-175, 1972.

M. Drton and M.D. Perlman. Multiple testing and error control in Gaussian graphical model selection. Statist. Sci., 22:430, 2007.

B. Efron, T. Hastie, I. Johnstone, and R. Tibshirani. Least angle regression. Ann. Statist., 32(2):407-499, 2004.

A Juditsky and A. Nemirovsky. On verifiable sufficient conditions for sparse signal recovery via $\ell_{1}$ minimization. ArXiv, 2008. URL http://arxiv.org/ abs/0809.2650.

K. Knight and W. Fu. Asymptotics for lasso-type estimators. Ann. Statist., 28 (5):1356-1378, 2000.

S. Lèbre. Inferring dynamic genetic networks with low order independencies. Statistical Applications in Genetics and Molecular Biology, 8(1), 2009.

B. Marlin, M. Schmidt, and K. Murphy. Group sparse priors for cavariance estimation. In Uncertainty in Artificial Intelligence, 2009.

N. Meinshausen and P. Bühlmann. High-dimensional graphs and variable selection with the lasso. Ann. Statist., 34(3):1436-1462, 2006.

N. Meinshausen and B. Yu. Lasso-type recovery of sparse representations for high-dimensional data. Annals of statistics, 2008.

R. Opgen-Rhein and K. Strimmer. Learning causal networks from systems biology time course data: an effective model selection procedure for the vector autoregressive model. BMC Bioinformatics, 8, 2007.

M.R. Osborne, B. Presnell, and B.A. Turlach. On the LASSO and its dual. J. Comput. Graph. Statist., 9(2):319-337, 2000.

M. Ronen, R. Rosenberg, B. Shraiman, and U. Alon. Assigning numberes to the arrows: parametrizing a gene regulation network by using accurate expression kinetics. PNAS, 99(16):10555-10560, 2002. 
J. Schäfer and K. Strimmer. A shrinkage approach to large-scale covariance matrix estimation and implications for functional genomics. Statistical Applications in Genetics and Molecular Biology, 4(1), 2005.

T. Shimamura, S. Imoto, R. Yamaguchi, and S. Miyano. Weighted lasso in graphical gaussian modeling for large gene network estimation based on microarray data. Genome Informatics, 19:142 - 153, 2007.

T. Shimamura, S. Imoto, R. Yamaguchi, A. Fujita, M. Nagasaki, and S. Miyano. Recursive regularization for inferring gene networks from time-course gene expression profiles. BMC Systems Biology, 3(41), 2009.

P.T. Spellman, G. Sherlock, M.Q. Zhang, V.R. Iyer, M.B. Eisen, P. Braow, D. Botstein, and B. Futcher. Comprehensive identification of cell cycleregulated genes of the yeast saccharomyces cerevisiae by microarray hybridization. Molecular Biology of the cell, 9:3273-3297, 1998.

R. Tibshirani. Regression shrinkage and selection via the lasso. J. Roy. Statist. Soc. Ser. B, 58(1):267-288, 1996.

A. Wille and P. Bühlmann. Low-order conditional independence graphs for inferring genetic networks. Statistical Applications in Genetics and Molecular Biology, 5(1), 2006.

M. Yuan and Y. Lin. Model selection and estimation in regression with grouped variables. Journal of the Royal Statistical Society, Series B, 68(1):49-67, 2006.

M. Yuan and Y. Lin. Model selection and estimation in the Gaussian graphical model. Biometrika, 94(1):19-35, 2007.

P. Zhao and B. Yu. On model selection consistency of lasso. Journal of Machine learning Research, 7:2541-2563, 2006.

S. Zhou, S. van de Geer, and P. Bühlmann. Adaptive lasso for high dimensional regression and Gaussian graphical modeling. ArXiv, 2009. URL http:// arxiv.org/abs/0903.2515v1.

H. Zou. The adaptive lasso and its oracle properties. J. Amer. Statist. Assoc., 101(476):1418-1429, 2006.

H. Zou and Hastie T. Regularization and variable selection via the elastic net. Journal of the Royal Statistical Society, 67:301-320, 2005.

H. Zou, T. Hastie, and R. Tibshirani. On the degrees of freedom of the lasso. Ann. Statist., 34(5):2173-2192, 2007. 